BMJ Paediatrics Open

\title{
Comparative efficacy and safety of interventions for treating head lice: a protocol for systematic review and network meta-analysis
}

Bill Stevenson, ${ }^{1}$ Wubshet Tesfaye, ${ }^{1}$ Julia Christenson, ${ }^{1}$ Cynthia Mathew, ${ }^{1}$ Solomon Abrha, ${ }^{1}$ Gregory Peterson, ${ }^{1,2}$ Indira Samarawickrema, ${ }^{1}$ Jackson Thomas (1) ${ }^{1}$

To cite: Stevenson B, Tesfaye W, Christenson J, et al. Comparative efficacy and safety of interventions for treating head lice: a protocol for systematic review and network meta-analysis. BMJ Paediatrics Open 2021;5:e001129. doi:10.1136/ bmjpo-2021-001129

- Additional online supplemental material is published online only. To view, please visit the journal online (http://dx.doi.org/10.1136/ bmjpo-2021-001129).

Received 15 April 2021 Revised 22 April 2021 Accepted 24 April 2021
Check for updates

\section{(C) Author(s) (or their} employer(s)) 2021. Re-use permitted under CC BY-NC. No commercial re-use. See rights and permissions. Published by BMJ.

${ }^{1}$ Faculty of Health, University of Canberra, Canberra, Australian Capital Territory, Australia ${ }^{2}$ Pharmacy, University of Tasmania, Hobart, Tasmania, Australia

\section{Correspondence to} Dr Jackson Thomas; Jackson. Thomas@canberra.edu.au

\section{ABSTRACT}

Background Head lice infestation is a major public health problem around the globe. Its treatment is challenging due to product failures resulting from rapidly emerging resistance to existing treatments, incorrect treatment applications and misdiagnosis. Various head lice treatments with different mechanism of action have been developed and explored over the years, with limited report on systematic assessments of their efficacy and safety. This work aims to present a robust evidence summarising the interventions used in head lice.

Method This is a systematic review and network meta-analysis which will be reported in accordance with the Preferred Reporting Items for Systematic Reviews and Meta-analyses statement for network meta-analyses. Selected databases, including PubMed, Embase, MEDLINE, Web of Science, CINAHL and Cochrane Central Register of Controlled Trials will be systematically searched for randomised controlled trials exploring head lice treatments. Searches will be limited to trials published in English from database inception till 2021. Grey literature will be identified through Open Grey, AHRQ, Grey Literature Report, Grey Matters, ClinicalTrials.gov, WHO International Clinical Trials Registry and International Standard Randomised Controlled Trials Number registry. Additional studies will be sought from reference lists of included studies. Study screening, selection, data extraction and assessment of methodological quality will be undertaken by two independent reviewers, with disagreements resolved via a third reviewer. The primary outcome measure is the relative risk of cure at 7 and 14 days postinitial treatment. Secondary outcome measures may include adverse drug events, ovicidal activity, treatment compliance and acceptability, and reinfestation. Information from direct and indirect evidence will be used to generate the effect sizes (relative risk) to compare the efficacy and safety of individual head lice treatments against a common comparator (placebo and/or permethrin). Risk of bias assessment will be undertaken by two independent reviewers using the Cochrane Risk of Bias tool and the certainty of evidence assessed using the Grading of Recommendations, Assessment, Development and Evaluations guideline for network

\section{What is already known on this topic?}

Head lice infestation is a significant public health problem affecting people irrespective of socioeconomic backgrounds.

- Several interventions including insecticides, occlusive agents and physical methods are available for head lice treatment.

\section{What this study hopes to add?}

- Despite the presence of several interventions, there is no reliable comparative data on the relative efficacy and safety of the interventions.

- It is not well established whether the dose, formulation, number of treatments and duration of head lice interventions have any meaningful impact on treatment outcomes.

- There is no strong evidence on the ovicidal activity of existing treatments.

meta-analysis. All quantitative analyses will be conducted using STATA V.16.

Discussion The evidence generated from this systematic review and meta-analysis is intended for use in evidence-driven treatment of head lice infestations and will be instrumental in informing health professionals, public health practitioners and policy-makers.

PROSPERO registration number CRD42017073375.

\section{BACKGROUND}

Head lice infestation (Pediculus humanus capitis) is a global public health issue that affects people of all socioeconomic backgrounds. ${ }^{1-3}$ Although there is a lack of reliable data, the prevalence estimates of head lice infestations in school-aged children range from $5 \%$ in Europe to $33 \%$ in Central and South America. ${ }^{4}$ Head lice infestations affect people regardless of ethnicity and age, although it is more common among children 
Table 1 A partial summary of treatments for head lice, adapted from Diamantis et al ${ }^{48}$

\begin{tabular}{|c|c|c|}
\hline Agents & Mechanism of action & Common adverse effects; limitations \\
\hline Pyrethrins & $\begin{array}{l}\text { Sodium channel blocker, neurotoxic and leads to } \\
\text { paralysis and death of the parasite }\end{array}$ & Local irritation, allergy \\
\hline Permethrin & $\begin{array}{l}\text { Synthetic drug similar to pyrethrins. Sodium channel } \\
\text { blocker as above. }\end{array}$ & Local irritation, allergy \\
\hline Fine combs & $\begin{array}{l}\text { Used for manual physical removal, often with } \\
\text { conditioners. }\end{array}$ & $\begin{array}{l}\text { Limited efficacy alone, repeat treatments are } \\
\text { often required }\end{array}$ \\
\hline
\end{tabular}

aged 7-14 years, females and vulnerable populations dwelling in crowded environments. ${ }^{156}$ Head lice infestation causes parental anxiety and acts as a source of economic loss through missed school days and caregiver time off work ${ }^{7}$ - the annual cost in the US alone was estimated at more than US $\$ 1$ billion. ${ }^{8}$

Head lice causes considerable discomfort, and intensive itching that could lead to poor sleep and excoriation-although uncommon, skin breaches superinfected with resistant pathogenic bacteria can lead to secondary skin infections and lymphadenopathies. ${ }^{9} 10$ Also, affected children and their parents often suffer from social stigma, embarrassment and low self-esteem, ${ }^{11} 12$ and some jurisdictions prevent children with head lice from attending schools altogether, resulting in school absenteeism and economic loss through caregiver absence from work. ${ }^{13} 14$ Consequently, governments dedicate a relatively large amount of resources to develop new products and to design strategies for the control and prevention head lice.

There are a range of interventions available for the management of head lice (table 1 ). ${ }^{15}$ The main stay of therapy has been largely dependent on insecticidal-based approaches for several decades. However, the accumulating evidence with resistance to frontline insecticidal treatments like pyrethrins, permethrin and malathion has led to a growing incentive to develop newer and more effective treatments to treat the condition safely. ${ }^{1}$ Over the past couple of decades, alternative candidates have been introduced into the market, including ivermectin, ${ }^{16-18}$ occlusive agents (eg, benzyl alcohol, isopropyl myristate and dimethicone) ${ }^{19-21}$ and herbal products ${ }^{22}$ and essential oils. ${ }^{22-25}$ While drugs with novel mode of action may potentially tackle the rapidly growing issue of resistance, in the absence of strong comparative evidence, the relative efficacy and safety of the newer agents and how they fare with insecticidal treatments remain unclear.

In addition to the variety of treatments, the significantly varied trial formats, and conflicting reviews from preceding decades make treatment choice in head lice management difficult. ${ }^{26}$ This has, in turn, caused a degree of ambiguity in terms of the relative efficacy and safety of currently existing head lice treatments. ${ }^{26}$ Further, owing to the increasing resistance to conventional treatments, there is a high incidence of treatment failure. ${ }^{28}$ Low effectiveness of the insecticidal products and the unproven nature of herbal products have led to parents resorting to dangerous alternatives, such as kerosene or veterinary flea products, in a desperate attempt to cure recalcitrant head lice infestations. Several studies $^{29-31}$ reported safety issues with many commonly used head lice treatments ranging from local itchiness ${ }^{30}$ to severe neurological conditions. ${ }^{31}$ While there is some literature on the subject, ${ }^{32}$ to our knowledge, there is lack of robust comparative evidence on the efficacy and safety of current treatments. ${ }^{11}$ Therefore, there is a critical need to evaluate the effectiveness and the safety profile of current head lice treatments to make evidenceinformed recommendations for health practitioners and wider community and thereby minimise the burden of head lice.

Between 1990 and 2001, only two major systematic reviews were conducted examining the effect of head lice treatments, both of these studies had substantial methodological limitations and reported inconclusive findings. ${ }^{26}$ Vander Stichele $e t a l^{33}$ concluded that only permethrin had enough evidence in terms of efficacy to justify its use in head lice treatment. In contrast, the Cochrane review performed by $\operatorname{Dodd}^{34}$ (published in 1999, and then revised in 2001) concluded that permethrin, malathion and pyrethrins were all effective treatments for head lice, although this conclusion relied on only three clinical trials that met their inclusion criteria. In 2006, the review by Dodd was withdrawn pending major substantive update-an updated study protocol that has since been, in turn, withdrawn in 2018. ${ }^{34}$ There is a 2019 systematic review that compared occlusive and neurotoxic head lice treatments as a group but not as individual treatments. ${ }^{35}$ It has been many years since a comprehensive systematic review and meta-analysis has been performed comparing individual head lice treatments. ${ }^{36}$ With Dodd review withdrawn and the review by Vander Stichele et al 25 years old, recent evidence on head lice treatment is clearly wanting. ${ }^{33}$ 
This systematic review and network meta-analysis seeks to bridge this gap and aims to generate conclusive evidence about the efficacy and safety of head lice interventions, with a view to inform clinicians, policy-makers and the public to guide them in their efforts to improve the quality of head lice treatment in the community.

\section{METHODS}

This study protocol is prepared in accordance with the Preferred Reporting Items for Systematic Reviews and Meta-analyses Protocols (PRISMA) ${ }^{37}$ and has been registered with the International Prospective Register of Systematic Reviews (PROSPERO; CRD42019132524).

\section{Patient and public involvement}

This protocol was designed without patient or public involvement.

\section{Search strategy, keywords}

Preliminary searches of the literature will be conducted to identify the keywords, which will be integrated and listed to undertake a more extensive search. These will be combined with Boolean operators and medical subject headings to formulate a refined search method. To identify the keywords regarding head lice interventions, the key words 'pediculos*', 'pediculus humanus capitis', 'head lice', 'clinical trials', 'pediculicide*', insecticide*, 'shampoo', 'conditioner' 'randomised control trials', 'controlled treatment studies', 'disease management', 'interventions', 'permethrin', 'ivermectin', 'malathion', 'pyrethrins', 'human', 'phytotherapy', 'essential oil*', 'drug therapy' OR clinical OR routine OR 'pharmaceutical preparations' OR 'treatment' OR 'intervention' OR 'therapy' OR 'medicine' OR ' management' will be used. Clinical trials published till 2021 will be considered for inclusion. Both completed clinical trials and trials in progress will be considered. The search strategy will be developed based on the instructions in the Cochrane handbook for systematic reviews of interventions ${ }^{38}$ and will be specific for each database. A detailed search strategy for PubMed, Embase via Scopus and Web of Science is shown in online supplemental appendix 1 . The results will be presented in accordance with the PRISMA flow chart.

\section{Information databases}

Electronic databases, including PubMed, Medline, the National Institute of Health and National Library of Medicine, SCOPUS, the Excerpt Medica Database (EMBASE), JURN and Google Scholar, will be searched for studies, along with the US clinical trials database (https://clinicaltrials.gov/), the Australia New Zealand Clinical Trial Registry http://www.anzctr.org.au/), the International Standard Randomised Controlled Trials Number (https://www.isrctn.com/) and the WHO's International Clinical Trials Registry Platform (https://www.who.int/ ictrp/en/). In addition, grey literature will be explored via Informit, the OaIster database and the WHO. Further, the references of the included articles will also be thoroughly screened for relevant articles potentially missed during the main search.

\section{Types of studies to be included}

Only primary randomised controlled trials (RCTs) that are published from inception of targeted databases until 2021 will be included based on the following criteria: participants of any age with live head lice or lice and eggs (not eggs alone) before enrolment studies with either placebo or active comparator group; and reported the outcomes of interest (pediculicidal and ovicidal activities).

\section{Participants/population}

The review will consider studies that include participants of any age, gender or country of origin. Exclusion criteria include participants with other similar lice (such as pediculus humanus or 'body lice') and participants with inactive infections (characterised by no live adult or nymph lice and eggs $>2 \mathrm{~cm}$ away from the scalp, if present).

\section{Intervention(s), exposure(s)}

Any RCT investigating treatment for head lice will be included, regardless of the nature of the intervention. Such treatments may include insecticides, suffocation products, essential oils, desiccants, deterrents or pediculicides. As such, any intervention designed with the intent of reducing or curing an extant head lice infection will be considered in this review. Exceptions include treatments exclusively designed for ovicidal effects.

\section{Comparator(s)/control}

Dependent on each trial, any comparator may be used. This may be between active therapeutic agents or between an active agent and a placebo.

\section{Outcome(s)}

The main outcome is cure from an active infestation measured as proportion of participants being completely free from head lice after 7 days postlast treatment or within 14 days postfirst treatment. If a trial evaluated outcomes in days other than 7 or 14 days, this will be assigned to the standard time frame where appropriate (eg; an outcome assessed on days $6,8,9$ or 10 will be assigned to day 7 , while outcomes assessed on days 11-21 would be assigned as day 14). Secondary outcomes that will be considered in this review are ovicidal activity of drugs, adverse events, treatment compliance and acceptability, and reinfestation, whenever reported.

\section{Selection of studies}

The retrieved citations will be transferred to Covidence ${ }^{39}$ for screening, data extraction and risk of bias (RoB) assessment. Full texts of potentially relevant studies that pass initial screening will be examined for eligibility. Where possible, reviewers will attempt to contact primary authors via email when eligibility for inclusion is not 
clear. Two independent reviewers will perform eligibility assessment, data extraction, and RoB assessment using the Covidence electronic platform. Any disagreements will be resolved by a third reviewer. Reasons for excluding studies/trials will be recorded.

\section{Data extraction}

This systematic review will be reported in accordance with the recommendations of the PRISMA statement for network meta-analyses. ${ }^{40}$ Two review authors will extract the data and check for discrepancies at each level (title, abstract and full text) using the inclusion and exclusion criteria. We will specifically extract the following data:

- General information: author, journal, year(s) the study took place, year of publication, country, sample size, sociodemographic characteristics of study participants; attrition and their characteristics.

- Study methodology: study design, inclusion/exclusion criteria, sample size.

- Details of intervention: dose, formulation, frequency and mode of application, duration of administration.

- Comparators: details of the comparator groupplacebo, no treatment, control intervention of nonpharmacological nature or other medicine.

- Outcomes: clinical cure following the study intervention, secondary outcomes and results (including effect estimates; adverse effects, acceptability).

- Study limitations.

- User satisfaction/preference of studied treatments.

Where applicable, data extracted will also include: other secondary outcomes reported by the studies and not listed in this review, reasons for patient removal from the trial, major advantages or disadvantages identified in the trial and the method by which the primary outcome was measured (ie, how was the presence or absence of adult lice determined). Data will be tabulated and sorted by treatment for further analysis. If the primary outcome data are missing, the authors of relevant studies will be contacted (if possible) to supply missing information. If the data cannot be obtained, the study will be excluded.

\section{Strategy for data synthesis}

Data will be collected by at least two reviewers independently and manually extracted from the selected papers. Discrepancy and disagreement will be discussed between reviewers when they occur until a consensus is reached. Key values will then be entered in a spreadsheet used by all reviewers. Once information has been extracted, it will be synthesised via a narrative approach and interpreted accordingly. Where possible, data will be tabulated for ease of access and readability.

A network meta-analysis will be performed based on the intention to treat population using a multivariate meta-analysis with consistency model, ${ }^{41}$ with restricted maximum likelihood (REML) estimation applied to calculate the pooled RRs across studies, with the findings presented alongside the narrative interpretation of the data. This REML model is preferred given it is unlikely to underestimate the variance like maximum likelihood estimation does. In terms of assessing between-study variations, the analysis assumes an exchangeable covariance structure at 0.5 . Information from direct and indirect evidence will be used to generate the effect sizes (relative risk) for each treatment against a common comparator (placebo or permethrin). The efficacy and safety of the different head lice treatments will be ranked using the surface under the cumulative ranking curve, which shows the percentage efficacy and safety of individual treatment against a hypothetically ideal treatment. Interventions will be considered at the level of an individual drug and outcome data for multiple doses or dosage formulations of a given intervention will be merged under a single treatment node. The agreement between estimates from direct and indirect evidence will be assessed using a design-by-treatment interaction model. ${ }^{42}$ The hypothesis of inconsistency will be assessed by globally testing all inconsistency parameters using a global Wald test statistic, ${ }^{43}$ while publication bias will be assessed using comparison-adjusted funnel plots. ${ }^{44}$

All quantitative analyses will be conducted using STATA V.16 (StataCorp) and a two-sided $\mathrm{p}<0.05$ will be used to show statistical significance.

\section{RoB assessment and grading of evidence}

The Cochrane RoB 2 (RoB-2) ${ }^{45}$ tool will be used to assess RoB in included studies, focusing on biases related to five key domains: randomisation process, deviations from intended interventions, missing outcome data, outcome measures and selection of the reported result. Each domain will receive a judgement on the RoB (high, low or some concerns) and an overall RoB will be assigned based on the judgements from the five domains. Each of two reviewers will independently apply the tool to each paper to determine its bias category, then confer with other reviewer. Any disagreements between the reviewers will be resolved by discussion with a third reviewer.

We will use the Grading of Recommendations Assessment, Development and Evaluation approach for network meta-analysis ${ }^{46}$ to report the quality of evidence on the efficacy and acceptability of different interventions to be included in the systematic review. The rate of quality for direct and indirect evidence will be performed separately, which will then be used to rate the quality of the network meta-analysis. The quality of evidence associated with direct comparisons will be assessed based on five key domains (methodology quality, the directness of evidence, heterogeneity, the precision of effect estimates and risk of publication bias). Given only randomised trials will be included, all the studies in the quality assessment will start from high and then be downrated for the reasons mentioned. In the case of the indirect evidence, factors like intransitivity (based on narrative comparisons of study characteristics) and network coherence (based on differences in direct and indirect effect estimates) will be used to assess the quality of evidence. Finally, the quality of evidence for the network will consider the 
direct and indirect evidence for the inventions and will be reported as high, moderate, low and very low.

\section{DISCUSSION}

This systematic review and network meta-analysis of head lice treatments is a comprehensive work aiming to provide a strong evidence base to understand the relative efficacy and safety of existing head lice interventions. By considering and comparing the evidence on important attributes of investigated drugs, including efficacy, safety and tolerability, ease of application, and cost, the reviewers will attempt to make clinical recommendations for head lice treatment. Furthermore, this work is anticipated to highlight the gaps in existing research on head lice treatments and illuminate the way forward. The last major systematic review in this area was published nearly two decades ago only to be withdrawn soon after. This work will provide consolidated, decisive evidence to inform clinicians on the best choice of intervention to treat a head lice infestation. However, due to the inclusion of RCTs only, we may miss on important rare adverse events associated with the treatments, which would have been observed in large-scale cohort studies.

\section{Twitter Wubshet Tesfaye @WubeHT}

Contributors BS, WT and JT conceived the idea. BS, WT, SA, CM, IS and GP contributed to the drafting and editing of the manuscript. All authors agreed on the final version of the manuscript.

Funding The authors have not declared a specific grant for this research from any funding agency in the public, commercial or not-for-profit sectors.

Competing interests None declared.

Patient consent for publication Not required

Ethics approval Ethics approval will not be required given the published works are available in the public domain. The findings from this systematic review will be communicated to the appropriate audience through conference abstract/s and a peer-reviewed journal publication.

Provenance and peer review Not commissioned; externally peer reviewed.

Supplemental material This content has been supplied by the author(s). It has not been vetted by BMJ Publishing Group Limited (BMJ) and may not have been peer-reviewed. Any opinions or recommendations discussed are solely those of the author(s) and are not endorsed by BMJ. BMJ disclaims all liability and responsibility arising from any reliance placed on the content. Where the content includes any translated material, BMJ does not warrant the accuracy and reliability of the translations (including but not limited to local regulations, clinical guidelines, terminology, drug names and drug dosages), and is not responsible for any error and/or omissions arising from translation and adaptation or otherwise.

Open access This is an open access article distributed in accordance with the Creative Commons Attribution Non Commercial (CC BY-NC 4.0) license, which permits others to distribute, remix, adapt, build upon this work non-commercially, and license their derivative works on different terms, provided the original work is properly cited, appropriate credit is given, any changes made indicated, and the use is non-commercial. See: http://creativecommons.org/licenses/by-nc/4.0/.

ORCID iD

Jackson Thomas http://orcid.org/0000-0002-0699-788X

\section{REFERENCES}

1 Falagas ME, Matthaiou DK, Rafailidis PI, et al. Worldwide prevalence of head lice. Emerg Infect Dis 2008;14:1493-4.

2 Burgess IF. Head lice. BMJ Clin Evid 2011;2011. [Epub ahead of print: 16 May 2011].
3 Burgess IF, Silverston P. Head lice. BMJ Clin Evid 2015;2015. [Epub ahead of print: 14 Jan 2015].

4 Hatam-Nahavandi K, Ahmadpour E, Pashazadeh F, et al. Pediculosis capitis among school-age students worldwide as an emerging public health concern: a systematic review and meta-analysis of past five decades. Parasitol Res 2020;119:3125-43.

5 Speare R, Buettner PG. Head lice in pupils of a primary school in Australia and implications for control. Int $J$ Dermatol 1999;38:285-90.

6 Monheit BM, Norris MM. Is combing the answer to headlice? J Sch Health 1986;56:158.

7 Frankowski BL, Bocchini JA, Council on School Health and Committee on Infectious Diseases. Head lice. Pediatrics 2010;126:392-403.

8 McCormack PL. Spinosad: in pediculosis capitis. Am J Clin Dermatol 2011;12:349-53.

9 Mumcuoglu KY, Klaus S, Kafka D, et al. Clinical observations related to head lice infestation. J Am Acad Dermatol 1991;25:248-51.

10 Roberts RJ. Head lice. N Engl J Med Overseas Ed 2002;346:1645-50.

11 Hurst SK, Dotson JAW, Butterfield P, et al. Stigma resulting from head lice infestation: a concept analysis and implications for public health. Nurs Forum 2020;55:252-8.

12 Parison JC, Speare R, Canyon DV. Head lice: the feelings people have. Int J Dermatol 2013:52:169-71.

13 Frankowski BL, Bocchini JA, CoS H, Council on School Health and Committee on Infectious Diseases. Head lice. Pediatrics 2010;126:392-403.

14 Gordon SC. Shared vulnerability: a theory of caring for children with persistent head lice. J Sch Nurs 2007;23:283-92.

15 Moosazadeh M, Afshari M, Keianian H, et al. Prevalence of head lice infestation and its associated factors among primary school students in Iran: a systematic review and meta-analysis. Osong Public Health Res Perspect 2015;6:346-56.

16 Chosidow O, Giraudeau B, Cottrell J, et al. Oral ivermectin versus malathion lotion for difficult-to-treat head lice. N Engl J Med 2010;362:896-905

17 Meinking TL, Mertz-Rivera K, Villar ME, et al. Assessment of the safety and efficacy of three concentrations of topical ivermectin lotion as a treatment for head lice infestation. Int J Dermatol 2013;52:106-12.

18 Pariser DM, Meinking TL, Bell M, et al. Topical 0.5\% ivermectin lotion for treatment of head lice. N Engl J Med 2012;367:1687-93.

19 Burgess IF, Brunton ER, Burgess NA. Single application of $4 \%$ dimeticone liquid gel versus two applications of $1 \%$ permethrin creme rinse for treatment of head louse infestation: a randomised controlled trial. BMC Dermatol 2013;13:5.

20 Burgess IF, Lee PN, Brown CM. Randomised, controlled, parallel group clinical trials to evaluate the efficacy of isopropyl myristate/ cyclomethicone solution against head lice. Pharmaceutical Journal 2008;280:371-5.

21 Meinking TL, Villar ME, Vicaria M, et al. The clinical trials supporting benzyl alcohol lotion 5\% (Ulesfia): a safe and effective topical treatment for head lice (pediculosis humanus capitis). Pediatr Dermatol 2010;27:19-24.

22 Williams CR, Webb CE, Orre S, et al. Can kunzea oil (Kunzea ambigua) control head lice (Pediculus humanus capitis)? Parasitology Open 2016;2:e3.

23 Di Campli E, Di Bartolomeo S, Delli Pizzi P, et al. Activity of tea tree oil and nerolidol alone or in combination against Pediculus capitis (head lice) and its eggs. Parasitol Res 2012;111:1985-92.

24 Greive KA, Lui AH, Barnes TM, et al. Safety and efficacy of a non-pesticide-based head lice treatment: results of a randomised comparative trial in children. Australas J Dermatol 2012;53:255-63.

25 Grieve K, Altman P, Rowe S, et al. A randomised, double-blind, comparative efficacy trial of three head lice treatment options: malathion, pyrethrins with piperonyl butoxide and MOOV head lice solution. Aust Pharmacist 2007;26:738-43.

26 Lapeere H, Vander Stichele RH, Naeyaert J-M. Evidence in the treatment of head lice: drowning in a swamp of reviews. Clin Infect Dis 2003;37:1580-2.

27 Sweileh WM. Global output of research on epidermal parasitic skin diseases from 1967 to 2017. Infect Dis Poverty 2018;7:74.

28 Downs AM, Stafford KA, Harvey I, et al. Evidence for double resistance to permethrin and malathion in head lice. $\mathrm{Br} J$ Dermatol 1999;141:508-11.

29 Clore ER, Longyear LA. A comparative study of seven pediculicides and their packaged nit removal combs. J Pediatr Health Care 1993;7:55-60.

30 Shuster J. Too much vitamin A lindane-induced CNS effects. Hosp Pharm 1996;31. 
31 Hammond K, Leikin JB. Topical pyrethrin toxicity leading to acuteonset stuttering in a toddler. Am J Ther 2008;15:323-4.

32 Burkhart CG. Relationship of treatment-resistant head lice to the safety and efficacy of pediculicides. Mayo Clin Proc 2004;79:661-6.

33 Vander Stichele RH, Dezeure EM, Bogaert MG. Systematic review of clinical efficacy of topical treatments for head lice. BMJ 1995;311:604-8.

34 Dodd CS. Interventions for treating headlice. Cochrane Database Syst Rev 2001;2:CD001165.

35 Flores-Genuino RNS, Gnilo CMS, Dofitas BL. Occlusive versus neurotoxic agents for topical treatment of head lice infestation: a systematic review and meta-analysis. Pediatr Dermatol 2020;37:86-92.

36 Do-Pham G, Le Cleach L, Giraudeau B, et al. Designing randomizedcontrolled trials to improve head-louse treatment: systematic review using a vignette-based method. J Invest Dermatol 2014;134:628-34

37 Moher D, Shamseer L, Clarke M, et al. Preferred reporting items for systematic review and meta-analysis protocols (PRISMA-P) 2015 statement. Syst Rev 2015;4:1.

38 Higgins J, Thomas J, Cochrane Community. Cochrane Handbook for systematic reviews of interventions, 2019. Available: https://training. cochrane.org/handbook/current

39 Covidence. Cochrane community, 2020. Available: https://www. covidence.org/reviewers [Accessed 23 Jun 2020].
40 Hutton B, Salanti G, Caldwell DM, et al. The PRISMA extension statement for reporting of systematic reviews incorporating network meta-analyses of health care interventions: checklist and explanations. Ann Intern Med 2015;162:777-84.

41 White IR. Multivariate Random-effects meta-analysis. Stata J 2009;9:40-56.

42 Higgins JPT, Jackson D, Barrett JK, et al. Consistency and inconsistency in network meta-analysis: concepts and models for multi-arm studies. Res Synth Methods 2012;3:98-110.

43 White IR, Barrett JK, Jackson D, et al. Consistency and inconsistency in network meta-analysis: model estimation using multivariate meta-regression. Res Synth Methods 2012;3:111-25.

44 Chaimani A, Higgins JPT, Mavridis D, et al. Graphical tools for network meta-analysis in STATA. PLoS One 2013;8:e76654.

45 Sterne JAC, Savović J, Page MJ, et al. RoB 2: a revised tool for assessing risk of bias in randomised trials. BMJ 2019;366:14898.

46 Puhan MA, Schünemann HJ, Murad MH, et al. A GRADE Working group approach for rating the quality of treatment effect estimates from network meta-analysis. BMJ 2014;349:g5630.

47 Brignardello-Petersen R, Florez ID, Izcovich A, et al. GRADE approach to drawing conclusions from a network meta-analysis using a minimally contextualised framework. BMJ 2020;371:m3900.

48 Diamantis SA, Morrell DS, Burkhart CN. Treatment of head lice. Dermatol Ther 2009;22:273-8. 